\title{
Sicherheitswirtschaft: Auch eine Public Management-Herausforderung
}

\author{
James W. Davis*
}

\begin{abstract}
The intersection between Security, Economics, and Technology has been under-researched and under-theorised leaving important questions unanswered in a world of growing, rapid change. This article begins to articulate the complex interrelationships between globalisation, the political economy of security, research and technology in order to better highlight how these changes in the global sphere are transforming national security policy into a commercially supplied product. As the private security industry increasingly influences public security provision, innovative forms of cooperation will be required to create a strategy for the globalisation of security that will benefit all.
\end{abstract}

Keywords: Globalisierung, globale Produktion, Privatwirtschaft, Sicherheitswirtschaft

\section{Einleitung ${ }^{1}$}

$\mathrm{S}$ eit über einem Jahrzehnt stellt der fortschreitende Globalisierungsprozess eine Herausforderung für die Politik, die Industrie und die Wissenschaft dar. Durch Globalisierung werden neue Formen der politischen und ökonomischen Organisation ermöglicht, die wiederum neue Chancen, aber auch Gefahren für herkömmliche Akteure bergen. Die weltweite Ausdehnung des Marktes und die zunehmenden grenzüberschreitenden Aktivitäten von Unternehmen, Nichtregierungsorganisationen und (friedlichen, aber auch gewalttätigen) transnationalen Netzwerken erfordern neue Strategien für die Privatwirtschaft sowie die öffentliche Verwaltung, die sich ihrerseits in vielen Bereichen einem zunehmenden Macht- und Autoritätsverlust gegenübersieht. Nicht zuletzt, weil durch die Ausdehnung eines globalen Marktes die Steuerungsmöglichkeiten der Staaten abhanden kommen, beschäftigen sich heute zahlreiche Wissenschaftler mit der Frage, wie unter diesen Voraussetzungen eine effektive Global Governance möglich ist.

Bisher hat sich die Diskussion über die Ursachen und Auswirkungen der Globalisierung jedoch fast ausschließlich mit den nichtmilitärischen Dimensionen dieses Phänomens beschäftigt. Ein Blick auf die mediale Berichterstattung der vergangenen Jahre gibt aber Grund zu der Annahme, dass die Globalisierung in gravierendem Maße auch die Sicherheitswirtschaft betrifft und wichtige politische, ökonomische sowie militärstrategische Fragen aufwirft, die bislang weder systematisch erfasst noch erforscht sind. Nicht zuletzt, um diese Forschungslücke zu schließen, hat das Institut für Politikwissenschaft der Universität St. Gallen Anfang 2007 als eine der ersten europäischen universitären Einrichtungen ein »Kompetenzzentrum für Sicherheitswirtschaft und Technologie« (SiWiTec) gegründet, das sich insbesondere mit den konzeptionellen und organisatorischen Aspekten von staatlich garantierter Sicherheit und den Folgen der Globalisierung der Sicherheitswirtschaft auseinandersetzen soll.

\footnotetext{
* Prof. James W. Davis, Ph.D., ist Professor für Internationale Beziehungen und Direktor des Instituts für Politikwissenschaft an der Universität St. Gallen.

1 Für ihre Hilfe und Kritik bedanke ich mich bei Ursula Jasper und Kuno Schedler.
}

Wie notwendig grundlegende Forschung in diesem Bereich ist, macht folgendes Beispiel deutlich, das die Verwicklung politischer, ökonomischer sowie militärischer Interessen in der gegenwärtigen Sicherheitswirtschaft belegt. Durch versteckte Zukäufe hat die vom russischen Staat gelenkte Wneschtorgbank in den vergangenen Jahren ihren Anteil an der European Aeronautic Defence and Space Company (EADS) auf rund sieben Prozent ausgebaut. Das Unternehmen EADS, das zum Teil ein politisches Projekt der Regierungen Frankreichs, Deutschlands und Spaniens darstellt, ist einer der wichtigsten europäischen Hersteller von Kampfjets, Hubschraubern, Satelliten und Lenkflugkörpern. Angesichts der Wneschtorgbank-Investitionen kursiert in politischen Kreisen nun die Frage, mit welcher Absicht der russische Staat, der in letzter Zeit wiederholt seine Öl- und Gasvorkommen zu politischen Zwecken instrumentalisiert hat, in die westeuropäische Sicherheitsindustrie einsteigen will. Damit verbunden ist zudem die Überlegung, wie sich die an EADS beteiligten Regierungen sowie die Unternehmensführung in Zukunft gegenüber solchen Investitionen verhalten sollen?

Die Reichweite der Probleme, die an der Schnittstelle der Bereiche Sicherheit, Wirtschaft und Technologie liegen, ist in der Tat groß. Da sich alle drei Bereiche in einer Phase des rapiden Wandels befinden, entstehen sowohl für die Wissenschaft als auch die politische Praxis bzw. die öffentliche Verwaltung spannende, aber auch herausfordernde Fragen.

Im Folgenden soll deshalb versucht werden, das Forschungsfeld Sicherheitswirtschaft und einige der Fragen, die durch die gegenwärtigen Entwicklungen virulent werden, kurz zu skizzieren. Die Darstellung ist eher illustrativ und keineswegs erschöpfend. Dennoch soll sie zeigen, dass tradierte Annahmen bezüglich des Sicherheitsverhaltens der Nationalstaaten hinterfragt und neue Strategien zur Gewährleistung der Sicherheit entwickelt werden müssen. Wenn eine der Hauptaufgaben des Staates immer noch die Herstellung von Sicherheit ist, dann müssen politische Entscheidungsträger nicht nur die rapiden Veränderungen auf dem globalen Markt zur Kenntnis nehmen, sondern neue Managementinstrumente entwickeln, um das gewollte Produkt Sicherheit und die dazu notwendigen Voraussetzungen nachhaltig bereitzustellen. 


\section{Sicherheit und Ökonomie}

Obwohl die bisher einflussreichste Denkschule der wissenschaftlichen Auseinandersetzung mit Aspekten nationaler oder internationaler Sicherheit, der politische Realismus, durch ihre deutliche Trennung zwischen der Sicherheitspolitik (high politics) einerseits und dem Streben nach Wohlstand (low politics) andererseits gekennzeichnet ist, war die Unterscheidung nie ganz unproblematisch. Zumindest seit dem 17. Jahrhundert argumentieren Merkantilisten, dass Wohlstand eine notwendige Voraussetzung von Macht sei; dass Macht essentiell für die Herstellung von Wohlstand sei; dass sowohl Macht als auch Wohlstand angemessene Ziele der Politik seien; und dass langfristig eine Harmonie zwischen den beiden Zielen bestehe. ${ }^{2}$ Auch Hans Morgenthau und Kenneth Waltz bezeichneten, als sie ihre jeweiligen Theorien der internationalen Politik entwickelten, ökonomische Stärke als eine Eigenschaft von Großmächten. ${ }^{3}$ Ferner lag der hegemonialen Ordnung, von der Robert Gilpin schrieb, eine durch Wirtschaft ermöglichte militärische Macht zu Grunde. ${ }^{4}$

In den spezialisierteren Bereichen der Sicherheitspolitik bzw. der Strategic Studies, welche sich erst in der Nachkriegszeit entwickelten, wurden jedoch die Verbindungen zwischen Sicherheit und Wirtschaft nur selten explizit thematisiert. Während des Kalten Kriegs verschaffte sich der spätere USVerteidigungsminister James Schlesinger mit seinem Buch The Political Economy of National Security, in dem er die Bedeutung politischer Entscheidungen für die Wirtschaftskraft und somit auch für die geostrategische Position der Vereinigten Staaten im Wettkampf mit der Sowjetunion charakterisierte, Profil. ${ }^{5}$ Gegen Ende des Ost-West Konflikts erschien zudem Ethan Kapsteins scharfsinnige Analyse unter dem gleichen Titel. Zwar war Anfang der 1990er Jahre das Wort »Globalisierung « relativ neu, jedoch argumentierte Kapstein bereits damals, dass die Sicherheitspolitik von den Veränderungen im Weltmarkt nicht unberührt bleiben werde. ${ }^{6}$ In der Regel aber beschränkten sich Analysten nach dem 2. Weltkrieg und während des Ost-West Konflikts auf die Analyse ökonomischer Mittel der allgemeinen Außenpolitik bzw. der »Staatskunst «. ${ }^{7}$

Festzustellen ist auch, dass sich in den letzten fünfzehn Jahren nur wenige Wissenschaftler systematisch mit dem Zusammenhang zwischen einerseits den Erfordernissen der nationalen und internationalen Sicherheit und andererseits den rapiden Entwicklungen auf dem globalen Markt, die unter dem Be-

2 Siehe Jacob Viner, Power versus Plenty as Objectives of Foreign Policy in the Seventeenth and Eighteenth Centuries, in: World Politics 1/1948, S. 1-29, insbesondere S. 10 .

3 Hans J. Morgenthau, Politics Among Nations: The Struggle for Power and Peace, 3. Edition (New York: Knopf, 1966), S. 113-118; Kenneth N. Waltz Theory of International Politics (New York: Random House, 1979), S. 131.

4 Robert Gilpin, War and Change in World Politics (Cambridge: Cambridge University Press, 1981), S. 122-155.

5 James R. Schlesinger, The Political Economy of National Security. A Study of the Economic Aspects of the Contemporary Power Struggle (New York: Praeger, 1960).

6 Ethan B. Kapstein, The Political Economy of National Security. A Global Perspective (New York: McGraw Hill, 1991). Siehe auch Helen V. Milner und David A. Baldwin, The Political Economy of National Security: An Annotated Bibliography (New York: Westview, 1990).

7 Zwei der besten Werke waren Albert O. Hirschman, National Power and the Structure of Foreign Trade (Berkeley: University of California Press, 1945); und später David A. Baldwin, Economic Statecraft (Princeton: Princeton University Press, 1986). griff »Globalisierung « verlaufen, auseinandersetzten. ${ }^{8}$ Wenn aber zutrifft, dass die materiellen und wirtschaftlichen Quellen nationaler Macht immer von Bedeutung waren und dass diese nun zunehmend von Prozessen der Globalisierung beeinträchtigt sind, dann muss das Thema Sicherheitswirtschaft eine zentrale Stelle sowohl in der politischen Praxis als auch in der Politikwissenschaft einnehmen.

\section{Effizienz oder Versorgungsabsicherung?}

Doch auch wenn Macht und Wohlstand sich gegenseitig bestimmen und verstärken, so sagt dies zunächst noch nichts über die Herstellung beider aus. Sobald man sich mit der Frage nach der richtigen Strategie zur Maximierung der Sicherheit eines Landes beschäftigt, wird man mit einem grundlegenden Dilemma, dem »Ressourcen-Beschaffungs-Dilemma«, konfrontiert. Es besteht darin, dass eine nationale Sicherheitspolitik gleichzeitig zwei Ziele zu maximieren versucht, die nicht gleichzeitig zu maximieren sind.

Zum einen will der Staat Effizienz maximieren. Das heißt, er will das Maximum an Sicherheit herstellen, welches das ihm zur Verfügung stehende Budget erlaubt. Zum anderen will er sicherstellen, dass die Versorgung mit den für die Schaffung von Sicherheit notwendigen Mitteln nicht durch politische oder ökonomische Störungen beeinträchtigt wird. Zwei unterschiedliche wirtschafts- und industriepolitische Strategien sind denkbar: Eine nationalistische Wirtschaftsstrategie, die konzipiert ist, durch Subventionen, Zölle und außertarifliche Handelshemmnisse ausländische Wettbewerbskonkurrenz herauszufiltern und nationale Unternehmen des Verteidigungssektors zu stärken, könnte sicherstellen, dass eine nationale verteidigungsindustrielle Basis existiert, die, wenn notwendig, nationale Sicherheitsanstrengungen unterstützt. Andererseits könnte ein solches Vorgehen aber auch zu wirtschaftlichen Verzerrungen, mangelnder Wettbewerbsfähigkeit und nicht zuletzt zu Ineffizienz führen, die wiederum den staatlichen Wohlstand und letztendlich die angestrebten Ziele nationaler Sicherheit gefährden könnten.

Eine wirtschaftsliberale Strategie hingegen, die auf den Fundamenten der freien Marktwirtschaft, der Spezialisierung und der Schaffung komparativer Vorteile fußt, hat demgegenüber den Vorteil der Maximierung des nationalen Wohlstands und der aggregierten Ressourcen, welche wiederum vom Staat für die nationale Verteidigung eingesetzt werden können. Nichtsdestoweniger hat eine solche Strategie unter Umständen den Effekt, dass (nicht-konkurrenzfähige) nationale Industrien aus dem Markt gedrängt werden, was wiederum bedeutet, dass der Staat auf diese indigenen Industrien und die von ihnen produzierten Sicherheitstechnologien nicht zurückgreifen kann, wenn er von internationalen Märkten abgeschnitten sein sollte. ${ }^{9}$

\footnotetext{
$8 \mathrm{Zu}$ den Ausnahmen gehören die Analysen in Jean-Marc F. Blanchard, Norrin M. Ripsman and Edward D. Mansfield (Hrsg), Power and the Purse: Economic Statecraft, Interdependence, and National Security (London: Routledge, 2000); Victor D. Cha, Globalization and the Study of International Security, in: Journal of Peace Research 3/2000, S. 391-403; und vor allem Stephen G. Brooks, Producing Security: Mulitnational Corporations, Globalization, and the Changing Calculus of Conflict (Princeton: Princeton University Press, 2005).

9 Norrin M. Ripsman, The Political Economy of Security: A Research and Teaching Agenda, in: Journal of Military and Strategic Studies: The Electronic Journal of the Centre for Military and Strategic Studies, (Spring 2000), S. 2.
} 
Bemerkenswert ist, dass seit der industriellen Revolution, kein Staat - nicht einmal die Vereinigten Staaten oder die Sowjetunion - innerhalb seines Territoriums über alle notwendigen Ressourcen verfügte, um sich vollständig autark zu entwickeln. Die Bedeutung dieser Tatsache ist somit klar: Das RessourcenBeschaffungs-Dilemma ist nicht durch eine einfache Optimierungsformel aufzulösen, sondern es erfordert eine politische Entscheidung in Abwägung zweier miteinander konkurrierender Werte. Diese Entscheidungen rufen wiederum immer wieder neu zu bewältigende Folgeprobleme hervor.

\section{Globalisierung: Produktion statt Handel}

Die vorherige Diskussion zeigt, dass Staaten bei der Herstellung von Sicherheit immer mit den Vor- und Nachteilen wirtschaftlicher Interdependenz konfrontiert waren. Es war Aufgabe der politischen Entscheidungsträger, die Balance zwischen Eigenständigkeit und internationaler Marktintegration zu bestimmen. Als die Interdependenzen primär aus Handel in Rohstoffen und/oder Endprodukten bestanden, war die Frage ob Märkte national, regional oder international integriert waren, zunächst eine Sache der politisch gesetzten Rahmenbedingungen. ${ }^{10}$

Verstehen wir Globalisierung nun also nur als eine Zunahme in der Breite und Dichte grenzüberschreitender Handelsbeziehungen, erscheint der Prozess nicht unbedingt als etwas Neues. Gemessen am Wert des globalen Außenhandels als Teil des globalen Bruttosozialprodukts war die Globalisierung Anfang des 20. Jahrhunderts bereits weiter entwickelt als bis zum Ende des Jahrhunderts. ${ }^{11}$ Erst in den 1990er Jahren wurde das Niveau von 1914 wieder erreicht. ${ }^{12}$

Doch dieser Vergleich, so interessant er auch sein mag, kann Analysten in die Irre führen, da die Globalisierung des 21. Jahrhunderts nicht primär durch die zunehmende Breite und Dichte an zwischenstaatlichem Handel zu erfassen ist. Das Wesentliche an der neuen Globalisierung ist der stetig zunehmende grenzüberschreitende, zwischen- und innerbetriebliche Handel, der wiederum eine kontinuierlich zunehmende Öffnung und Zergliederung der Produktionsketten widerspiegelt. Nicht der globale Handel, sondern die globale Produktion stellt also das wahrhaft Revolutionäre an der heutigen Globalisierung dar. ${ }^{13}$

Die Globalisierung des Welthandels Anfang des 20. Jahrhunderts wurde durch eine erhebliche Senkung der Transportkosten ausgelöst. Durch die Entwicklung der Eisenbahn, des Dampfers und des Kraftfahrzeugs konnten Menschen und Waren relativ schnell und zu zahlbaren Preisen auch über die Grenzen des eignen Territoriums hinaus transportiert werden.

10 Stephen Kobrin, Regional Integration in a Globally Networked Economy, in: Transnational Corporations, 2/1995, S. 15-34.

11 Für die relevanten Daten siehe: Angus Maddison, Monitoring the World Economy (Paris: OECD, 1995).

12 Maurice Obstfeld und Alan M. Taylor, The Great Depression as a Watershed International Capital Mobility in the Long Run, Working Paper No. 5960 (Cambridge, MA: NBER, 1997), S. 1.

13 Siehe Peter Dicken, Global Shift: Transforming the World Economy, 3. Edition (New York: Guilford Press, 1998); Peter Dicken, Global Shift: Reshaping the Global Economic Map in the Twenty-First Century, 4. Edition (New York: Guilford Press, 2002); und Stephen Kobrin, The Architecture of Globalization: State Sovereignty in a Networked Global Economy, in: John Dunning (Hrsg.), Governments, Globalization, and International Business (Oxford: Oxford University Press, 1997).
Die heutige Globalisierung wird ebenfalls durch technologische Veränderungen vorangetrieben - die Transportkosten sind noch weiter gesunken. Aber es ist primär die dramatische Senkung der Informations- und Kommunikationskosten durch Digitalisierung, Glasfasertechnologie, das Internet usw. sowie der gleichzeitige Wandel weg von arbeits- und gebundenen kapitalintensiven Volkswirtschaften hin zu Wissensökonomien, die die Globalisierung der Produktion ermöglichen. ${ }^{14}$

Zusammengenommen erlauben diese Entwicklungen zum einen die Öffnung der Beschaffungskette nicht nur für weltweite Zulieferer der Roh- bzw. Verbrauchsstoffe eines Endprodukts, sondern auch jener Bauteile und Industrieerzeugnisse, die bereits einen großen Mehrwert und eine hohe Komplexität aufweisen. ${ }^{15}$ Bereits in den 1980er Jahren war das Verhältnis von importierten zu national-produzierten Bauteilen in Deutschland um 38 Prozent, in Japan um 40 Prozent, in Frankreich um 81 Prozent, in den USA um 86 Prozent und in Großbritannien um 131 Prozent gestiegen. ${ }^{16}$

Zum anderen macht dieser Prozess eine universale Produktionsstrategie innerhalb einer Firma möglich. Durch die weltweite Zerstreuung der Produktion schöpfen Firmen jene Effizienzen aus, die ortsgebunden sind. Und diese Entwicklung hin zu globaler Produktion ist keineswegs auf die größten Firmen beschränkt, wie eine Studie der Konferenz der Vereinten Nationen für Handel und Entwicklung belegt: »In today's globalizing world economy, the increasing competitive pressures faced by firms of all sizes impel more and more of them to establish an international portfolio of locational assets to remain competitive $\ll \cdot{ }^{17}$ Mittlerweile beträgt der Anteil des internationalen Welthandels, der aus innerbetrieblichem Austausch in Industrieerzeugnissen besteht, ein Drittel des Gesamtvolumens. ${ }^{18}$

Dass die dramatische Steigerung jenes Anteils am internationalen Austausch in Waren und Dienstleistung, der auf innerbetrieblichen Handel zurückzuführen ist, auch politische Konsequenzen erzeugen kann, hat Helen Milner bereits 1988 überzeugend dargelegt. Verblüfft vom Weiterbestand des Freihandelssystems während der Inflation und der schwachen Konjunktur der 1970er Jahre, entdeckte Milner das starke Interesse global integrierter Firmen am Erhalt niedriger Handelsbarrieren. Anders als früher forderten Firmen heute auch bei schwacher Konjunktur oder Inflation nicht den Schutz ihrer Märkte durch Zölle und Handelshemmnisse. Vielmehr sind sie, so Milners Argumentation, aufgrund des mittlerweile global organisierten innerbetrieblichen Handels an einem ungehinderten Warenfluss interessiert. ${ }^{19}$

14 Die Entwicklung von Containern und Supertankern etwa hat die realen Kosten des Seetransports zwischen Anfang der 1980er und Mitte der 1990er Jahre um 70 Prozent gesenkt. Die Kosten eines Telefongesprächs zwischen London und New York sind heute 1.500-mal billiger als im Jahre 1930! (Siehe Brooks, Producing Security, S. 27.)

15 Geoffrey Garrett, The Causes of Globalization,in: Comparative Political Studies 6-7/2000, S. 995.

16 Brooks, Producing Security, S. 26

17 United Nations Conference on Trade and Development (UNCTAD), World Investment Report, 1994: Transnational Corporations, Employment, and the Workplace (Geneva: United Nations, 1994), S. 4. Zitiert in Brooks, Producing Security, S. 27.

18 Jeffrey Jones, The Evolution of International Business. An Introduction. (New York: Routledge, 1996).

19 Helen V. Milner, Resisting Protectionism: Global Industries and the Politics of International Trade (Princeton: Princeton University Press, 1988). 
In der Zwischenzeit haben die Auswirkungen der Globalisierung der Produktion dazu geführt, dass Staaten mit hohen Opportunitätskosten rechnen müssen, falls sie sich von diesen Prozessen abschotten. ${ }^{20}$ Heute zeichnen Firmen mit globalintegrierter Produktion für drei Viertel der weltweiten Investitionen in Forschung und Entwicklung verantwortlich. Diese Firmen stellen nicht nur eine unverzichtbare Quelle neuer Technologien dar, sondern fördern durch ihre Schulungsaktivitäten weitere Innovationen in ihren Standorten. Staaten, die wirtschaftlich an vorderster Stelle landen oder bleiben wollen, sind somit gezwungen, nicht nur Handelshemmnisse, sondern auch Barrieren gegenüber ausländischen Direktinvestitionen zu senken, um die Aktivitäten global produzierender Firmen zu optimieren. So erzeugt die gegenwärtige, Technologie getriebene Globalisierung eine sich selbst verstärkende politische Dynamik. ${ }^{21}$

\section{Die globale Produktion nationaler Sicherheit}

Wie die vorangegangenen Überlegungen gezeigt haben, lässt sich die gegenwärtige Globalisierung von ähnlichen historischen Prozessen in erster Linie durch die Globalisierung der Produktion unterscheiden. Jedoch ist diese Entwicklung nicht in allen Industriesektoren zu beobachten. Vielmehr ist die internationale Öffnung der Produktionskette auf diejenigen Industrien konzentriert, die einen hohen Forschungs- und Entwicklungsaufwand und Degressionseffekte auf der Firmenebene aufweisen - z.B. Maschinenbau, Elektronik, IT, Chemie, Kunst- und Werkstofftechnik, sowie die Luft- und Raumfahrtindustrie. Zunehmend wird die Entwicklung zudem von der Entstehung zwischenbetrieblicher Allianzen (inter-firm alliances) oder durch Technologie-Kooperationsabkommen (technology cooperation agreements) begleitet - vor allem in denjenigen Sektoren, die durch rapide Technologieveränderungen und hohe Markzutrittsbarrieren charakterisiert sind. Es handelt sich dabei also auch um jene wichtigen Sektoren der Herstellung moderner Waffen-, Aufklärungs-, Kommunikations-, und Führungssysteme: das Rückgrat der Verteidigung.

Da heute die Herstellung von Sicherheit in einer historisch beispiellosen Art und Weise von Hightech-Industrien abhängig ist, sind die angesprochenen Entwicklungen in der globalen Produktion von zentraler Bedeutung für politische Entscheidungsträger. Streben Staaten nach den modernsten, für die nationale Sicherheit relevanten Systemen, dann müssen sie ihre Sicherheitswirtschaft für die Prozesse der globalen Produktion öffnen. Denn das Fazit der bisher weitreichendsten Studie der Rüstungsindustrie im Zeitalter der Globalisierung macht deutlich: Kein Staat kann in der Militärtechnologie effektiv an vorderster Stelle bleiben, ohne eine Strategie der globalen Produktion zu verfolgen. ${ }^{22}$

20 Für eine Besprechung der Forschung zu dieser Frage, siehe Geoffrey Garrett, The Causes of Globalization. S. 960-967.

21 Brooks, Producing Security, S. 38-44. Das Argument ist nicht unumstritten. Siehe z.B. Dani Rodrik, Who needs capital-account convertibility?, in: S. Fischer et al. (Hrsg.), Should the IMF Pursue Capital Account Convertibility? Essays in International Finance 207 (Princeton. Department of Economics, Princeton University, 1998), S. 55-65; Eric Heilleiner, States and the Reemergence of Global Finance (Ithaca: Cornell University Press, 1994).

22 Brooks, Producing Security, S. 6.
Empirische Entwicklungen scheinen diese These zu bestätigen. Als die Rüstungsindustrie in den Vereinigten Staaten Mitte der 1970er Jahre eine Strategie der globalen Produktion und der zwischenbetrieblichen Allianzbildung einschlug, die in der breit diskutierten » Revolution in Military Affairs « mündete, ${ }^{23}$ verfolgte Brasilien, einst das im Bereich der Waffentechnologie fortgeschrittenste Exportland der Dritten Welt, noch eine Strategie der »technologischen Unabhängigkeit«. Das Resultat: Die brasilianische Rüstungsindustrie brach in den 1990er Jahre zusammen. ${ }^{24}$ Die Volksrepublik China hingegen, die bisher nicht in der Lage war, mit den Amerikanern und Japanern bei der Produktion von technologisch hoch entwickelten Waffensystemen mitzuhalten, hat in jüngster Zeit erste Schritte zur Öffnung der Rüstungsindustrie und zum Aufbau einer globalen Produktion unternommen. ${ }^{25}$

Was jedoch trotz all der offensichtlichen Vorteile einer internationalen Produktion hoch entwickelter Sicherheits- und Waffensysteme immer deutlicher zu Tage zu treten scheint, ist, dass sich nicht nur die strategische Handlungsfreiheit des Staates angesichts der Prozesse des globalen Marktes reduziert, sondern gleichzeitig die Anzahl der vom Staat zu bewältigenden Managementprobleme zunimmt.

Zunächst ist festzustellen, dass eine vollständige Absicherung bzw. Verteidigung der globalen Beschaffungskette hochkomplexer sicherheitsrelevanter Produkte unmöglich geworden ist. Um die Dimensionen der Herausforderung zu verdeutlichen, dient eine Studie des amerikanischen Handelsministeriums, derzufolge die Beschaffungsketten für drei Waffensysteme der Marine 15.000 Zulieferer nachweisen. ${ }^{26}$ Somit entsteht aus Sicht des Staates ein starker Anreiz, das Management der Produktion als Frage des öffentlichen, anstatt des privaten Sektors zu verstehen. Doch die steigende geographische Zerstreuung der Entwicklung und Produktion sicherheitsrelevanter Technologien, vor allem zivil und militärisch nutzbarer (dual-use) Systeme, bedeutet nicht nur eine Zunahme der Anzahl potenzieller Ziele eines feindlichen Angriffs. Sie bedeutet auch den Verlust des Monopols über Waffensysteme, auf das zumindest ein starker Staat bis in die 1980er Jahre bauen konnte. Zwar sind die modernsten Waffensysteme aufgrund ihrer enormen Komplexität nicht leicht in die Arsenale der Länder der Dritten Welt oder nichtstaatlicher Akteure wie Al-Qaida zu integrieren, doch ist der Zugang zu solchen Waffen somit durch die

23 Mit dem Begriff »Revolution in Military Affairs « ist die Einführung von Computern und Informationstechnologien in den Streitkräften gemeint, die zu einer Änderung der Kriegsführung und der Natur des Krieges führen soll. Präzisionslenkwaffen, Computernetzwerke und Echtzeit-Aufklärungssysteme sollen eine Kriegsführung von nie gekannter Präzision und Verlustfreiheit, für Freund und Feind, ermöglichen. Für eine zugängliche Einführung siehe: Elinor C. Sloan, The Revolution in Military Affairs (Montreal: M>GillQueen>s University Press, 2002).

24 Das Beispiel wird von Brooks erwähnt. Siehe Producing Security, S. 224. Siehe auch Ken Conca, Manufacturing Insecurity: The Rise and Fall of Brazil's Military-Industrial Complex (Boulder, CO: Lynne Rienner, 1997); und Ken Conca, Between Global Markets and Domestic Politics: Brazil's Military-Industrial Collapse, in: Review of International Studies 4/1998, S. 497-551.

25 John Wilson Lewis und Xue Litai, China's Search for a Modern Air Force, in: International Security 1/1999 S. 64-94; Evan A. Feigenbaum, Who's Behind China's High-Technology »Revolution«? How Bomb Makers Remade Beijing's Priorities, Policies and Institutions, in: International Security 1/1999, S. 99-126.

26 United States Department of Commerce, National Security Assessment of the Domestic and Foreign Subcontractor Base. A Study of Three U.S. Navy Weapons Systems (Washington, DC: Department of Commerce, 1992). 
Globalisierung der Produktion sowie durch den erleichterten Zugang zum Wissen dramatisch vereinfacht worden. ${ }^{27}$

Ein besonderes Gefahrenpotenzial liegt jedoch nicht nur in eben dieser Zergliederung der Produktionskette und in der globalen Ausbreitung von Dual-Use-Produkten, sondern auch in der Verschmelzung und Kooperation großer Konzerne innerhalb der sicherheitsrelevanten Sektoren: Während der 1980er Jahre entstanden 228 zwischenbetriebliche Allianzen in der Luftfahrt- und Verteidigungsindustrie und 60 Prozent aller Allianzen fanden in drei sicherheitskritischen Industrien - IT, Kunststofftechnik, und Chemie - statt. ${ }^{28}$ Angesichts der steigenden Konzentration in wichtigen Teilen der Rüstungsindustrie sowie einer steigenden Anzahl strategischer Allianzen und Technologie-Abkommen zwischen Firmen, die sich bisher im Sicherheitssektor als Konkurrenten gegenüberstanden, stellt sich die Frage, ob die Marktkonkurrenz vorhanden ist, die der Staat für eine wettbewerbliche Beschaffung von Verteidigungsmitteln benötigt. ${ }^{29}$ Im Extremfall kann es zur Substitution eines öffentlichen durch ein privates Monopol kommen, was wiederum zum Rent-Seeking führt, also zum Versuch, durch die Ausnutzung von Macht- und Informationsvorteilen einen Marktvorsprung zu erzielen. ${ }^{30}$ So stellt der effiziente Umgang mit Staatsmitteln vor dem Hintergrund dieser gegenwärtigen Entwicklung eine besondere Herausforderung für die öffentliche Verwaltung dar.

Vorstellbar ist auch, dass das Fehlen eines gesunden Marktmechanismus, bedingt durch die Marktkonzentration und eine wachsende Dichte globaler zwischenbetrieblicher Allianzen, zu einem erhöhten Einfluss der Industrie in sicherheitspolitischen Fragen führt. Es könnte beispielsweise - ohne in dieser Frage Position beziehen zu wollen - argumentiert werden, dass der Meinungswandel, der in der französischen Regierung mit Bezug auf das amerikanische Raketenabwehrsystem stattgefunden hat (von klarer Ablehnung hin zu wohlwollendem Schweigen), darauf hin deutet, dass es EADS durch konsequentes Lobbying und durch die enge Verzahnung mit der französischen Regierung gelungen ist, wirtschaftliche Interessen vor eine sicherheitspolitische Analyse zu stellen und somit auf die nationale und internationale Sicherheitspolitik einen deutlichen Einfluss zu nehmen.

\section{Akteursveränderung}

Die dargestellte Verschiebung bzw. das Aufweichen der Trennlinie zwischen den Bereichen des privaten und des öffentlichen Managements einerseits und der Sicherheitspolitik und Wirtschaftspolitik anderseits führen zu Veränderungen der Interessen von Staaten und Firmen. Sie wird auch von einer

27 Siehe z.B. Richard Falkenrath, Confronting Nuclear, Biological and Chemical Terrorism, in: Survival 3/1998, S. 43-65; Cha, Globalization and the Study of International Security, S. 396: John Arquilla und David Ronfeldt, The Advent of Netwar (Santa Monica, CA: RAND, 1996); Deborah Yarsike Ball und Theodore P. Gerber, Russian Scientists and Rogue States. Does Western Assistance Reduce the Proliferation Threat?, in: International Security 4/ 2005, S. 50-77.

28 Brooks, Producing Security, S. 85

29 Siehe hierzu John Alford und Deirdre O'Neill, The Contract State (Geelong: Deakin University Press, 1994), Kap. 4-5.

30 Kuno Schedler und Isabella Proeller, New Public Management (Bern: UTB, 2006), S. 48.
Veränderung der Identitäten der in den jeweiligen Sektoren tätigen Akteure begleitet. Zwar war der Markt immer schon vom Zusammenspiel zwischen Staaten und privaten Akteuren geprägt; die Gewährleistung von nationaler und internationaler Sicherheit war jedoch völkerrechtlich seit dem Westfälischen Frieden in der Regel dem Staat bzw. zwischenstaatlichen Institutionen vorbehalten. ${ }^{31}$ Auch wenn die Annahme, der Staat genieße ein Gewaltmonopol, selbst im modernen Staatensystem nie idealtypisch zutraf, fand eine historische Entwicklung weg von privaten und hin zu öffentlichen Sicherheitsakteuren statt. Beispielhaft hierfür waren die Reformen des 19. Jahrhunderts. Zumindest in der westlichen Welt gab es überall eine Reform der Heere, bei der Söldner und Adel durch Bürger ersetzt wurden. ${ }^{32}$ Im Zuge der Globalisierung gibt es jedoch Anzeichen einer Trendwende.

Weltweit ist die Privatwirtschaft heute zunehmend in die Herstellung von Sicherheit involviert, sei es innerhalb der territorialen Grenzen eines Landes oder im Ausland. Am sichtbarsten sind die sogenannten privaten Sicherheitsfirmen (private security companies), die Aufgaben erfüllen, die einst von Soldaten wahrgenommen wurden. In Afrika, zum Beispiel, ersetzen zahlreiche private Akteure fehlende militärische Fähigkeiten schwacher Staaten. ${ }^{33}$ Auch die führenden militärischen Kräfte verlagern immer mehr wichtige Aufgaben auf private Sicherheitsfirmen - eine Entwicklung, die durch die große Präsenz solcher Firmen während des Krieges gegen Saddam Hussein sowie der nachfolgenden Besetzung Iraks verdeutlicht wird. ${ }^{34}$ Firmen dieser Art bewegen sich mittlerweile auf einem globalen Markt, dessen Volumen auf 200 Milliarden US-Dollar ${ }^{35}$ geschätzt wird. Und sie betätigen sich dabei nicht nur im Kampfeinsatz; ihre Aktivitäten erstrecken sich von der Unterstützung von Führungssystemen (Command, Control, Communications, Computers and Intelligence - C4I) bis hin zur Logistik, Ausbildung und Planung. Zu ihren Kunden zählen nicht nur Staaten, sondern auch internationale Organisationen wie die Vereinten Nationen, NGOs oder private Firmen.

Auch wenn sich die meisten Studien über die Ursachen des Aufkommens privater Sicherheitsfirmen einig sind und dies auf die Ineffizienz des Staates zurückführen, so besteht dennoch keine Einigkeit über die Auswirkungen einer Verlagerung herkömmlicher Staatsaufgaben auf den privaten Sektor. Die einen ver-

31 Eine wichtige Ausnahme stellten die Aktivitäten privater Akteure in der kolonialen Welt dar. Siehe z.B. David Martin und Phyllis Johnson, The Struggle for Zimbabwe (Harare: Zimbabwe Publishing House, 1981); John Keay, The Honourable Company. A History of the English East India Company (London: Harper Collins, 1991).

32 Janice E. Thompson, Mercenaries, Pirates, and Sovereigns: State-Building and Extraterritorial Violence in Early Modern Europe (Princeton, NJ: Princeton University Press, 1994). Für eine Erklärung des Wandels siehe Deborah Avant, From Mercenary to Citizen Armies: Explaining Change in the Practice of War, in: International Organization 1/2000, S. 41-72.

33 Bernadette Muthien und Ian Taylor, The Return of the Dogs of War? The Privatization of Security in Africa, in: Rodney Bruce Hall und Thomas J. Biersteker (Hrsg.), The Emergence of Private Authority in Global Governance (Cambridge: Cambridge University Press, 2002), S. 183-199.

34 Auf der amerikanischen Seite waren zehn Prozent des Einsatzpersonals am Anfang des Krieges Angestellte von Privatfirmen. Im Mai 2004 schätzte US-Verteidigungsminister Donald Rumsfeld die Zahl der von privaten Sicherheitsfirmen angestellten Kräfte im Irak auf 20.000, die wiederum aus Ländern wie Fidschi, Israel, Nepal, Südafrika sowie den USA selbst stammten. Siehe Deborah Avant, Private Security Companies, in: New Political Economy 1/ 2005, S. 121-131.

35 Avant, Private Security Companies, S. 122. Siehe auch Larry Makinson, Outsourcing the Pentagon: Who Benefits from the Politics and Economics of National Security? (Washington, DC: Center for Public Integrity, 2004). 
sprechen sich verstärkte Fähigkeiten im Bereich Verteidigung und peacekeeping zum billigeren Preis, während andere Analysten davor warnen, dass durch die Auslagerung neue Kosten entstehen. ${ }^{36}$ Hinzu kommen eine Reihe politischer Fragen: Welche Auswirkung hat etwa eine Auslagerung staatlicher Aufgaben auf die Beziehungen zwischen der Exekutive und dem Parlament innerhalb demokratischer Staaten? Und welche Möglichkeiten bestehen, die Aktivitäten privater Akteure im Dienste der internationalen Sicherheit und der allgemeinen Menschenrechte durch nationales und Völkerrecht zu regeln?

\section{Fazit}

Bisher hat sich die Diskussion über die Ursachen und Auswirkungen der Globalisierung fast ausschließlich mit den

36 Zur Debatte siehe u.a. Eliot Cohen, Defending America's Interest in the Twenty-first Century, in: Foreign Affairs 6/2000, S. 40-56; David Shearer, Outsourcing War, in: Foreign Policy 112/1998, S. 68-82; Ken Silverstein, Private Warriors (London: Verso, 2000). nichtmilitärischen Dimensionen dieses Phänomens auseinandergesetzt. Der Prozess der Globalisierung und die damit einhergehende globale Zerstreuung der Produktion auch im Bereich sicherheitsrelevanter Industrien hat jedoch tiefgreifende Auswirkungen auf die Bereitstellung nationaler und internationaler Sicherheit: Die Trennlinie zwischen den Bereichen des privaten und des öffentlichen Managements einerseits und der Sicherheitspolitik und Wirtschaftspolitik andererseits wird zunehmend perforiert; gleichzeitig wächst der Einfluss der Privatwirtschaft auf die Bereitstellung von Sicherheit - nicht nur durch die Zunahme privater Sicherheitsfirmen, sondern auch durch Unternehmensfusionen, Zusammenschlüsse und Technologiekooperationen. Umso notwendiger erscheint es, dass sich Wissenschaft und Regierungen grundlegend mit der Frage auseinandersetzen, mit Hilfe welcher Strategien die Globalisierung der Sicherheitswirtschaft begleitet und für das Allgemeinwohl nutzbar gemacht werden kann.

\title{
Sicherheit als Geschäft: Der Aufstieg privater Sicherheits- und Militärunternehmen und die Folgen
}

\section{Gerhard Kümmel*}

\begin{abstract}
For quite some time already, violent international conflict has been influenced by a phenomenon that has come to be termed the privatization of security. The production and maintenance of security has increasingly been delegated to private enterprises by both state and non-state actors. Obviously, the business of security servicing is one that pays, as the war in Iraq impressively illustrates. The present paper looks at the root causes of the rise of a business whose growth potential is huge. At the same time this paper deals with the actual or potential consequences of this development. Ultimately, this paper concludes that private military and security companies are ambivalent in character. For governments as well as for other principals to employ these agents implies both advantage and risk, and thus strategies for a more discerning use of this instrument are in high demand.
\end{abstract}

Keywords: Privatisierung der Sicherheit, Private Sicherheits- und Militärunternehmen, Entstaatlichung der Sicherheit, Neue Kriege.

\section{Einleitung}

Anfang Juli dieses Jahres sorgte ein Bericht der Los Angeles Times für ein gewisses Aufsehen, legte er doch dar, dass die bislang genannten Zahlen von Privatkontraktoren im Konfliktherd Irak weit untertrieben und realiter um ein Mehrfaches höher waren als bislang angenommen. Diesem Zeitungsartikel liegen Informationen des State Department, der U.S. Agency for International Development (USAID) und des U.S. Central Command (CENTCOM) der amerikanischen Streitkräfte zugrunde, das vom amerikanischen Kongress bereits im Jahr 2006 dazu verpflichtet worden war, einen Privatkontraktoren-Zensus für den Irak zu erstellen. Danach verhält es sich so, dass "private contractors outnumber U.S. troops in Iraq«, stünden doch nach dem Stand vom

\footnotetext{
* Dr. Gerhard Kümmel ist Wissenschaftlicher Direktor am Sozialwissenschaftlichen Institut der Bundeswehr in Strausberg bei Berlin und Vorsitzender des Arbeitskreises Militär und Sozialwissenschaften (AMS). Der Autor vertritt in diesem Beitrag ausschließlich seine persönliche Ansicht.
}

Februar 2007 rund 160.000 US-Soldaten plus einigen wenigen Tausend zivilen Regierungsangestellten wenigstens 182.000 Privatkontraktoren und Privatsubkontraktoren gegenüber, die aus amerikanischen Steuergeldern finanziert würden, darunter etwa 21.000 Amerikaner, 43.000 Ausländer und 118.000 Iraker. Die Mehrzahl dieser rund 180.000 Privatkontraktoren, nämlich etwa 130.000 Personen, stünde dabei in den Diensten der amerikanischen Streitkräfte, mehrere Zehntausend würden von USAID beschäftigt, der Rest vom amerikanischen Außenministerium. ${ }^{1}$

Nun gehören beileibe nicht alle Privatkontraktoren, die in diese Gesamtsumme eingerechnet werden, zu den uns an dieser Stelle interessierenden Privatkontraktoren, nämlich den privaten Sicherheits- und Militärunternehmen (PSMU), die ihren diversen Kunden, zu denen Regierungen, internationale

\footnotetext{
1 T. Christian Miller: Private Contractors Outnumber U.S. Troops in Iraq. New U.S. Data Show How Heavily the Bush Administration Has Relied on Corporations to Carry out the Occupation of the War-Torn Nation. In: Los Angeles Times vom 4. Juli 2007.
} 